\title{
Surface- and tip-enhanced Raman spectroscopy of DNA
}

\author{
L.E. Hennemann, A.J. Meixner* and D. Zhang* \\ Institute of Physical and Theoretical Chemistry, University of Tübingen, Tübingen, Germany
}

\begin{abstract}
Calf thymus DNA adsorbed on a rough gold substrate or on an atomically smooth gold (111) surface has been investigated by collecting its unique Raman fingerprints using either surface-enhanced Raman scattering (SERS) or tip-enhanced Raman scattering (TERS). A monolayer coverage of DNA strands adsorbed at both the irregular rough edges of evaporated gold grids and at gold nanoparticles is detected by SERS. Highly improved sensitivity down to single DNA strand spectroscopic determination is accomplished by TERS providing an enhancement factor of at least 1400. Based on our experimental results, we propose that TERS is a promising technique to study the DNA-drug molecule interaction on the level of a single DNA strand.
\end{abstract}

Keywords: DNA, Raman spectroscopy, SERS, TERS, parabolic mirror

\section{Introduction}

Comprehension of biomolecular interaction is crucial for the development and improvement of drugs [10]. One of many examples is the interaction of the antibacterial, mutagenic and antitumoral drug 9-aminoacridine with DNA [6], the intercalation of which between the nucleic bases suppresses DNA replication at the binding site [4]. Spectroscopic methods such as Raman scattering or IR absorption have been applied to the determination of DNA genomic investigation without labelling. Raman spectroscopy provides a unique fingerprint of the molecules under investigation and is thus a highly appropriate tool for the study of biological samples. However, conventional Raman spectroscopy is an ensemble approach where locally and temporally restricted effects will get smeared out and weak signals will easily be covered by stronger ones. DNA-drug interactions give such subtle signals; for their study, more sensitive and selective methods are required that ideally measure on a single molecular level. Conventional Raman spectroscopy fails at such low concentrations because of the intrinsically low intensity of the inelastically scattered light. Surface-enhanced Raman scattering (SERS) or tip-enhanced Raman scattering (TERS) are two possible solutions to this problem.

SERS [3,12], relies on two commonly considered mechanisms: firstly, electromagnetic enhancement due to the resonant excitation of localised surface plasmons (LSP) of noble metal structures, and secondly the enhancement due to chemical effects such as charge-transfer complexes between the molecules and the substrate. Various SERS substrates have been investigated so far, both varying the noble metal (gold, silver or copper are usually used) and the shape of the plasmonic structures.

\footnotetext{
*Corresponding authors: Prof. Alfred J. Meixner and Dr. Dai Zhang, Institute of Physical and Theoretical Chemistry, University of Tübingen, Auf der Morgenstelle 8, 72076 Tübingen, Germany. E-mails: \{alfred.meixner, dai.zhang\}@unituebingen.de.
} 
TERS [1] is a fairly new spectroscopic technique. It makes use of the same fundamental principle of LSP excitation as SERS does; however, the geometry is turned upside down: Instead of providing a rough substrate below the molecules, the plasmonic mode is excited in a custom shaped noble metal tip which approaches the molecules from above. This tip is the crucial element that turns TERS into a highly versatile combination of Raman spectroscopy and topographical scanning probe microscopy. Atomic force microscopy (AFM) [2], scanning tunnelling microscopy (STM) [8] and shear force microscopy $[11,15]$ have all been employed successfully. The optical resolution obtained in TERS is determined by the size of the tip apex [7] and optical resolutions of 10-20 nm are achievable, a range far below the Rayleigh limit of conventional optical microscopy. The TERS effect makes it possible to study few or even single resonant molecules, as was shown by Steidtner et al. when measuring a single cresyl blue molecule [13]. In the field of biomolecular studies, Bailo et al. recently obtained highly reproducible TERS spectra along a single poly(cytosine) RNA strand [2].

In order to excite the tip, most TERS-setups illuminate the tip from below, i.e. through a transparent sample [2] or asymmetrically from the side [8]. However, the setup used for our investigations illuminates the tip symmetrically from above by the strongly longitudinally polarized focus of a parabolic mirror. With the gold tip in such a focus, the E-field of the light acts upon the free electrons of the metal, inducing dipole oscillations within the tip and along the tip axis. Further advantages are the large illumination and collection angle of this alignment and the fact that non-transparent samples such as noble metals can be used.

In this paper we will present both SERS and TERS measurements of DNA molecules performed with this custom built parabolic mirror based near field optical microscope. We will discuss SERS spectra where rough evaporated noble metal edges or gold nanoparticles yield the enhancement. We will show strongly enhanced TERS spectra of DNA strands adsorbed on an atomically flat gold (111) crystal and discuss the enhancement factor obtained.

\section{Experimental section}

The parabolic mirror based near-field optical microscope was already described previously $[9,14,15]$. A radially polarized donut beam [5] of a helium-neon laser $(\lambda=632.8 \mathrm{~nm})$ is focused on the sample surface. The power in the focus is $250 \mu \mathrm{W}$. The focusing is accomplished by a parabolic mirror of high numerical aperture $(N A=0.998)$. The focal field is in this case confined to a spot of only $260 \mathrm{~nm}$ in diameter and has a strong polarization perpendicular to the sample's surface.

The same parabolic mirror also acts as the signal-collecting element. The scattered light is separated from the incoming light through a beam splitter and then directed either to an avalanche photodiode (APD) or to a spectrometer coupled with a liquid nitrogen cooled CCD camera. Additionally, only the inelastically scattered Stokes shifted light is selected by means of two consecutively aligned notch filters.

SERS measurements were done in confocal far field mode, meaning that no tip was employed. Two means for providing the crucial surface roughness were used. In the first case, the rough edges of an evaporated gold grid on silicon were utilized. To produce these rough edges, $20 \mathrm{~nm}$ of gold were evaporated through a copper grid that was attached to a silicon surface. After removing the copper grid, the substrate now covered with a gold grid was put into a solution of calf thymus DNA $(1.7 \mathrm{mg} / \mathrm{ml})$ in triple distilled water. After 45 min adsorption time, the sample was rinsed carefully in order to remove DNA multilayers.

In the second case, a configuration of molecules sandwiched between an evaporated gold grid and gold nanoparticles of $20 \mathrm{~nm}$ diameter was created. The gold grid with adsorbed DNA molecules was 
prepared as described above. Subsequently, a $30 \mu$ volume of gold nanoparticles in solution was applied to the gold grid sample for $5 \mathrm{~min}$ and then spin-coated at $1600 \mathrm{r} / \mathrm{min}$ in order to spread the nanoparticles homogeneously on the surface.

For the TERS measurements, a fine gold tip was brought into the laser focus through a hole in the parabolic mirror. Glued to one prong of a quartz tuning fork, the tip is kept at a constant distance in the order of $2 \mathrm{~nm}$ via shear-force feedback control.

The tips were produced by electrochemical etching of gold wires of $100 \mu \mathrm{m}$ diameter in concentrated $\mathrm{HCl}$ solution. Tips with an apex of as small as about $20 \mathrm{~nm}$ diameter were obtained, leading to an optical resolution of the same scale [7]. All tips were investigated by scanning electron microscopy before use in order to make sure to only use well-shaped tips with similar apex curvatures.

The substrate used for the TERS measurements was a gold (111) crystal which was freshly annealed before every measurement. A DNA solution of $1.6 \mathrm{mg} / \mathrm{ml}$ calf thymus DNA in Millipore water was weakly sonicated for 5-10 s in order to untangle the DNA strands from each other. The crystal was immersed into this DNA solution for $2 \mathrm{~h}$ and then rinsed thoroughly in Millipore water to eliminate multilayers.

\section{Results and discussion}

A confocal photoluminescence image of the evaporated gold grid on silicon with additional gold nanoparticles spin-coated all over the sample is shown in Fig. 1a. The edges of the gold appear bright due to the random formation of gold clusters, some of which are resonantly excited by the $632.8 \mathrm{~nm}$ helium-neon laser. Further bright spots can be seen where the spin-coated nanoparticles are located on the closed gold film. These are the so-called "hot spots", where highly concentrated electric fields lead to locally enhanced photoluminescence. The inset in Fig. 1a shows a sketch of a DNA strand in such a "hot spot".
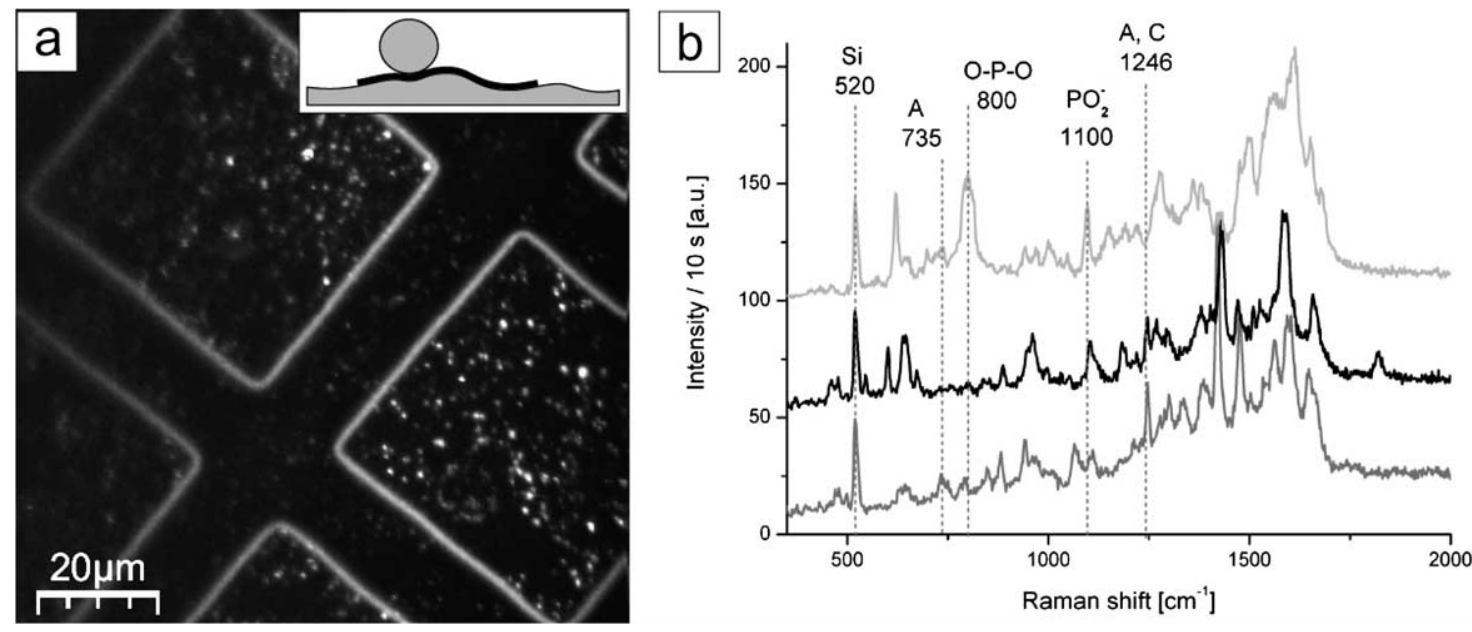

Fig. 1. SERS measurements of calf thymus DNA adsorbed on rough gold edges with additionally spin-coated gold nanoparticles. (a) Confocal optical image of the sample. The left-hand side looks dimmer due to a slight slope of the sample. Inset: sketch of a DNA strand in the optical "hot spot" between the evaporated gold and a gold nanoparticle. (b) Three SERS spectra of a (sub)monolayer of DNA molecules obtained in the configuration as sketched in (a). Exemplary, some of the Raman bands were assigned according to the literature values ( $\mathrm{A}$ - adenine, $\mathrm{T}$ - thymine, $\mathrm{G}$ - guanine, $\mathrm{C}$ - cytosine). Acquisition time: $10 \mathrm{~s}$, source: $632.8 \mathrm{~nm}$ helium-neon laser, laser power: $250 \mu \mathrm{W}$. 
On the rough gold edges, no Raman bands associable to DNA molecules were observed. Spectra obtained from the "hot spots" of the spin-coated nanoparticles are depicted in Fig. 1b. The strong Raman band at $520 \mathrm{~cm}^{-1}$ originates from the silicon substrate that produces a detectable signal even through $20 \mathrm{~nm}$ of evaporated gold film. The other bands show obvious fluctuations both in the intensities and the positions - a typical SERS feature of DNA molecules, especially when measuring close to the single molecule regime. Nevertheless, the bands can be assigned to literature values: among others there is the typical adenine band at $735 \mathrm{~cm}^{-1}$, the $\mathrm{O}-\mathrm{P}-\mathrm{O}$ vibrational stretching around $800 \mathrm{~cm}^{-1}$, the $\mathrm{PO}_{2}{ }^{-}$band at $1100 \mathrm{~cm}^{-1}$ and finally at $1246 \mathrm{~cm}^{-1}$ a band assigned to both adenine and cytosine.

The disadvantage of SERS is that the geometry leading to LSP cannot be tailored. Instead, one has to rely on random structures to give "hot spots" and on the random adsorption of the molecules in such "hot spots". Tip-enhanced Raman spectroscopy overcomes this problem: The near field source is now associated with the tip that scans the sample surface systematically. The location of DNA strands can be detected topographically, whereupon TERS spectra can be taken with accurate lateral control.

Results from our TERS measurements are depicted in Fig. 2. The topography of approximately a monolayer of calf thymus DNA strands recorded by shear force feedback can be seen in Fig. 2a. The inset sketches the configuration of a DNA strand located in the gap between the tip and its mirror image inside the gold (111) crystal. The tip acts as a very efficient optical antenna, channelling the electric field energy of the laser light into the near field arising in this gap. Such a "gap mode" configuration seizes the "hot spot" principle of SERS, however, in TERS the configuration is created in a controlled manner. The second inset is a topographical line cut showing the strands to be approximately $1 \mathrm{~nm}$ high.
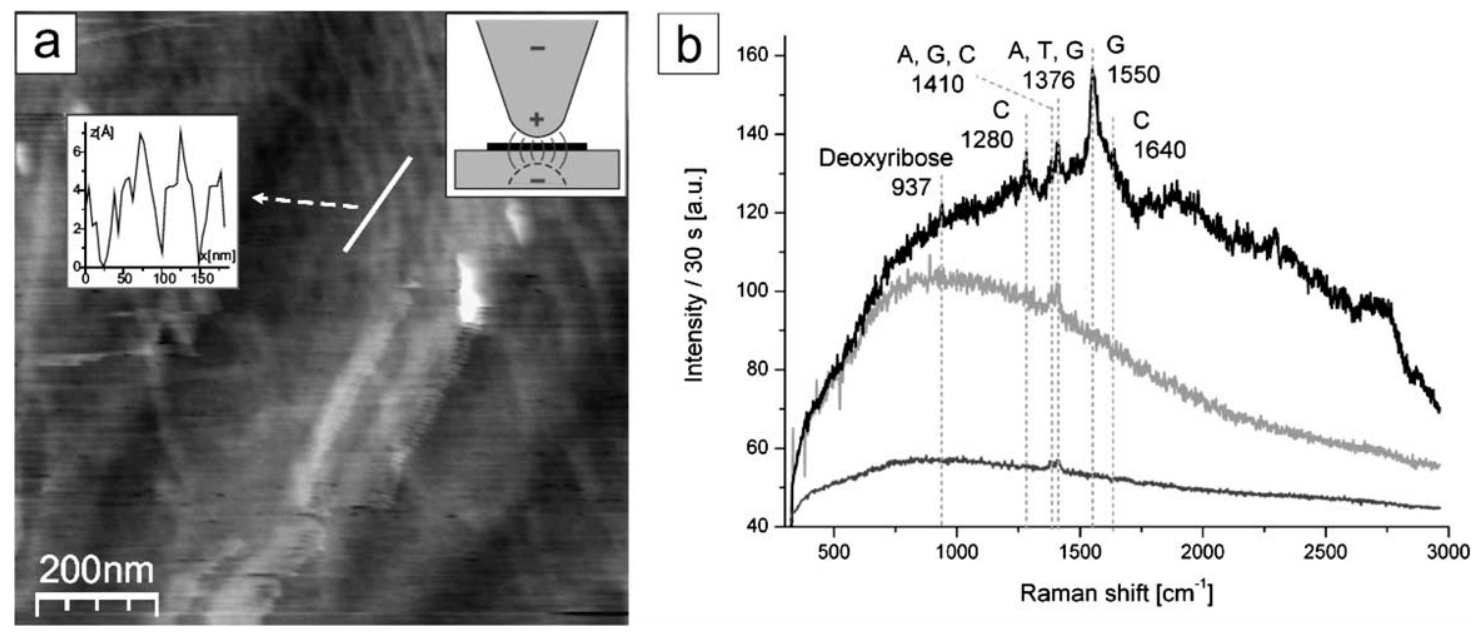

Fig. 2. TERS measurements of a (sub)monolayer of calf thymus DNA on a gold (111) crystal. (a) Shear force topographical image of the sample, including a line cut (inset graph). Sketched inset: Illustration of producing a "hot spot" in the gap between a fine gold tip and the substrate. The + and - signs symbolize the dipole arising inside the tip induced via excitation with a radially polarised donut beam. The curved vertical streaks represent the electrical field lines connecting the tip and its mirror charge inside the gold crystal. (b) Three Raman spectra taken consecutively at one specific spot on the sample. From bottom to top: confocal far field spectrum, acquisition time: $2 \mathrm{~min}$ but normalised to $30 \mathrm{~s}$; spectrum with the tip close to the surface, acquisition time: $30 \mathrm{~s}$; spectrum with the tip approached further to the surface, acquisition time: $30 \mathrm{~s}$. A tentative assignment of some of the Raman bands was undertaken (A - adenine, $\mathrm{T}$ - thymine, $\mathrm{G}$ - guanine, $\mathrm{C}$ - cytosine). Source: $632.8 \mathrm{~nm}$ helium-neon laser, laser power: $250 \mu \mathrm{W}$. 
Although DNA has a diameter of $2.37 \mathrm{~nm}$, this is in good agreement with previous measurements of DNA by AFM [11], since the DNA strands are slightly compressed by the force acting on them.

Figure $2 \mathrm{~b}$ shows three spectra obtained at the same spot. The lowermost spectrum was collected without tip, i.e. in confocal far field mode, with an acquisition time of $2 \mathrm{~min}$. A continuous background with a maximum around $900 \mathrm{~cm}^{-1}$ can be seen, arising from the photoluminescence of the gold crystal. Hardly any Raman bands rise above this background due to the lack of any Raman enhancement. The spectrum was normalised to an integration time of $30 \mathrm{~s}$ for better comparison with the next two spectra. These show from the middle to the top spectra collected upon approaching of the tip to the sample, obtained with an integration time of $30 \mathrm{~s}$. Compared to the confocal spectrum, we notice an increase of the gold photoluminescence. Upon further approaching the tip, the maximum of this background photoluminescence shifts to longer wavelengths. In addition, distinct Raman bands appear. They can tentatively be assigned to Raman bands of the DNA's bases and to its sugar.

The TERS enhancement factor can be calculated by $F_{\text {enh. }}=I_{\text {TERS }} \cdot A_{\text {conf. }} / I_{\text {conf. }} \cdot A_{\text {TERS }}$, where $I$ is the intensity of a specific Raman band (background subtracted) and A the area illuminated either by the confocal far field focus or by the TERS near field of the tip. We calculated the enhancement factor of this data set based on the 45.2 a.u. high TERS band at $1550 \mathrm{~cm}^{-1}$. Since in confocal far field mode this band does not appear, it has to be below the noise level of 0.775 a.u. The area excited in confocal far field mode is $5.31 \times 10^{-14} \mathrm{~m}^{2}$, deduced from our focal diameter of $260 \mathrm{~nm}$. With TERS, the area of excitation is given by the tip apex diameter. In the topographical image of this same measurement we observe the DNA strands to be $17 \mathrm{~nm}$ in diameter (see line cut in Fig. 2a), but these $17 \mathrm{~nm}$ are in fact a convolution of the tip apex and the much thinner DNA strands and thus give us directly the tip apex diameter. Hence, the TERS area is $2.27 \times 10^{-16} \mathrm{~m}^{2}$ and we can calculate the enhancement factor to be at least 14,000. Furthermore, given the topographical convolution of $17 \mathrm{~nm}$, we conclude that a fraction of one single DNA strand is excited by TERS in Fig. $2 b$.

\section{Conclusion and outlook}

This report has shown the successful use of parabolic mirror assisted confocal far field and near-field optical microscopy to the study of DNA strands. Especially spin-coating gold nanoparticles on top of a (sub)monolayer of calf thymus DNA shows very characteristic surface-enhanced Raman peaks at an integration time of merely $10 \mathrm{~s}$. Taking the step from the far field to the near-field mode and from SERS to TERS, DNA strands immobilized on a gold (111) crystal could be resolved by shear force scanning probe microscopy. On this substrate, a strongly enhanced TERS spectrum of a fraction of one single DNA strand was obtained with an integration time of $30 \mathrm{~s}$, which conceptually proves the possibility of investigating DNA-drug molecular interaction on a single DNA strand level. Further research about the calf thymus DNA-anti tumour drug interaction will be carried out and specific questions, such as the interaction property and interaction sites, will be emphasised on a few or single DNA strand level.

\section{Acknowledgements}

We acknowledge financial support from the Forschungsschwerpunktprogramm des Landes BadenWürttemberg and from the Projektförderung für NachwuchswissenschaftlerInnen an der Universität Tübingen (5577). 


\section{References}

[1] E. Bailo and V. Deckert, Tip-enhanced Raman scattering, Chemical Society Reviews 37 (2008), 921-930.

[2] E. Bailo and V. Deckert, Tip-enhanced Raman spectroscopy of single RNA strands: towards a novel direct-sequencing method, Angewandte Chemie International Edition 47 (2008), 1658-1661.

[3] C.L. Haynes, A.D. McFarland and R.P. van Duyne, Surface-enhanced Raman spectroscopy, Analytical Chemistry 77 (2005), 338A-346A.

[4] H. Ihmels and D. Otto, Intercalation of organic dye molecules into double-stranded DNA - general principles and recent developments, Supermolecular Dye Chemistry 258 (2005), 161-204.

[5] M.A. Lieb and A.J. Meixner, A high numerical aperture parabolic mirror as imaging device for confocal microscopy, Optics Express 8 (2001), 458-474.

[6] A. Murza, S. Alvarez-Mendez, S. Sanchez-Cortes and J.V. Garcia-Ramos, Interaction of antitumoral 9-aminoacridine drug with DNA and dextran sulfate studied by fluorescence and surface-enhanced Raman spectroscopy, Biopolymers 72 (2003), 174-184.

[7] L. Novotny, R.X. Bian and X.S. Xie, Theory of nanometric optical tweezers, Physical Review Letters 79 (1997), 645-648.

[8] B. Pettinger, K.F. Domke, D. Zhang, G. Picardi and R. Schuster, Tip-enhanced Raman scattering: influence of the tipsurface geometry on optical resonance and enhancement, Surface Science 603 (2009), 1335-1341.

[9] M. Sackrow, C. Stanciu, M.A. Lieb and A.J. Meixner, Imaging nanometre-sized hot spots on smooth au-films with highresolution tip-enhanced luminescence and Raman near-field optical microscopy, ChemPhysChem 9 (2008), 316-320.

[10] F. Sanger, S. Nicklen and A.R. Coulson, DNA sequencing with chain-terminating inhibitors, Proceedings of the National Academy of Sciences of the United States of America 74 (1977), 5463-5467.

[11] M. Sasou, S. Sugiyama, T. Yoshino and T. Ohtani, Molecular flat mica surface silanized with methyltrimethoxysilane for fixing and straightening DNA, Langmuir 19 (2003), 9845-9849.

[12] G.C. Schatz, M.A. Young and R.P. van Duyne, Electromagnetic mechanism of SERS, in: Surface-Enhanced Raman Scattering: Physics and Applications, Springer-Verlag, Berlin, Germany, 2006, pp. 19-45.

[13] J. Steidtner and B. Pettinger, Tip-enhanced Raman spectroscopy and microscopy on single dye molecules with $15 \mathrm{~nm}$ resolution, Physical Review Letters 100 (2008), 236101.

[14] X. Wang, D. Zhang, K. Braun, H.-J. Egelhaaf and A.J. Meixner, Confocal and near-field spectroscopic investigation of P3HT:PCBM organic blend film upon thermal annealing, Proceedings of SPIE 7395 (2009), 73950F.

[15] D. Zhang, X. Wang, K. Braun, H.-J. Egelhaaf, M. Fleischer, L. Hennemann, H. Hintz, C. Stanciu, C.J. Brabec, D.P. Kern and A.J. Meixner, Parabolic mirror-assisted tip-enhanced spectroscopic imaging for non-transparent materials, Journal of Raman Spectroscopy 40 (2009), 1371-1376. 


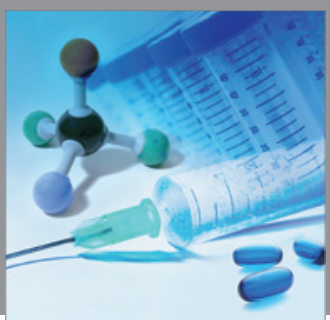

International Journal of

Medicinal Chemistry

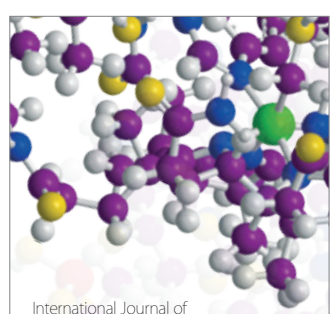

Carbohydrate Chemistry

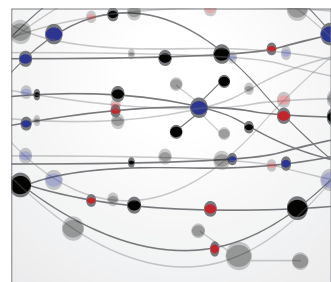

The Scientific World Journal
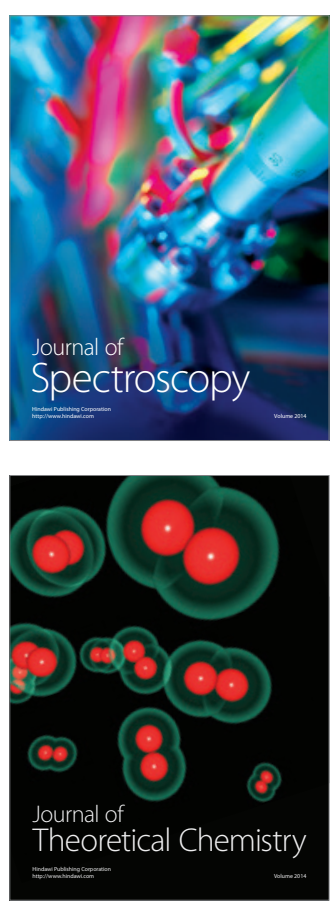
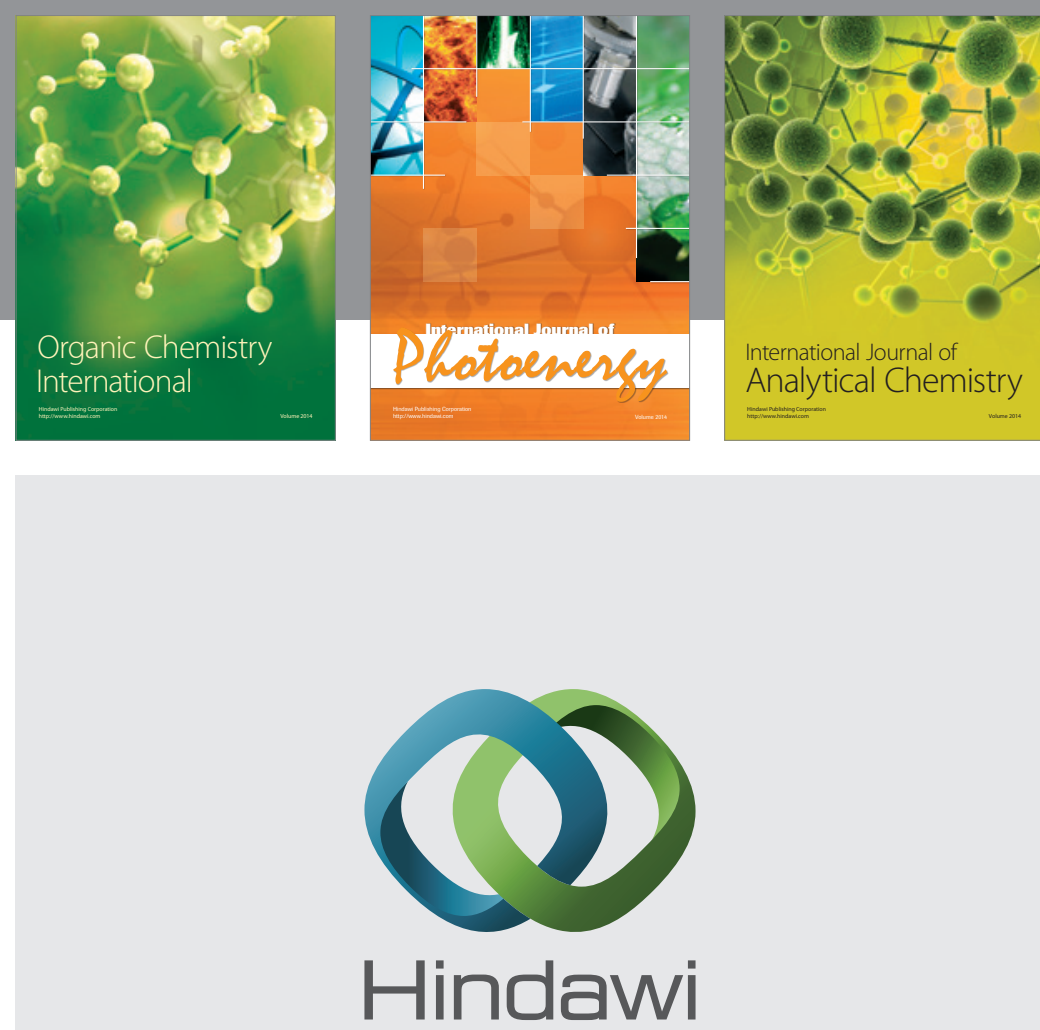

Submit your manuscripts at

http://www.hindawi.com
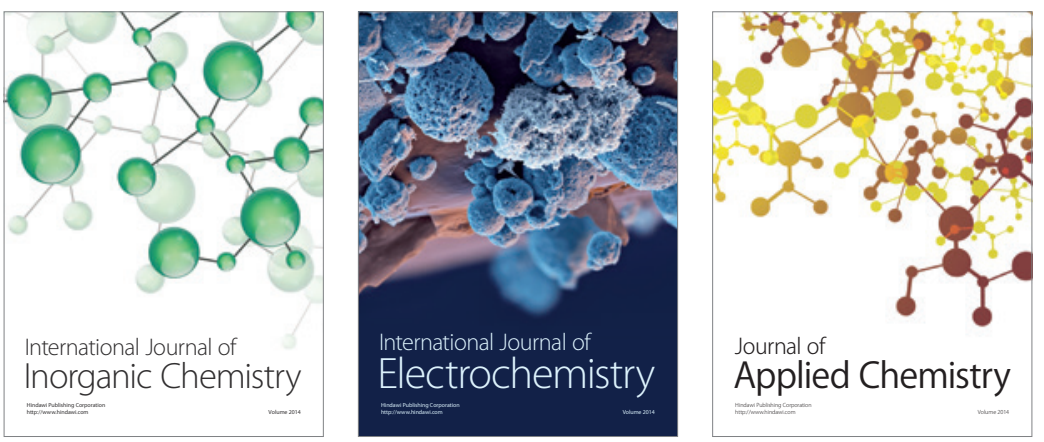

Journal of

Applied Chemistry
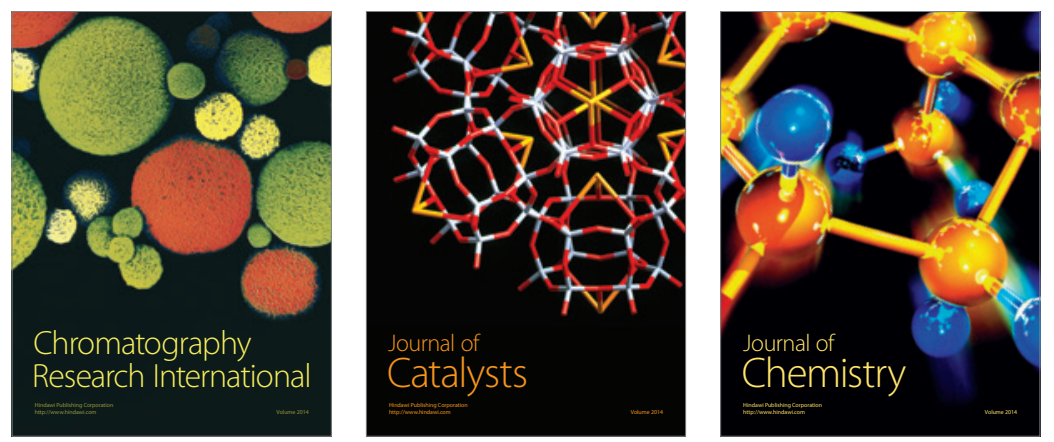
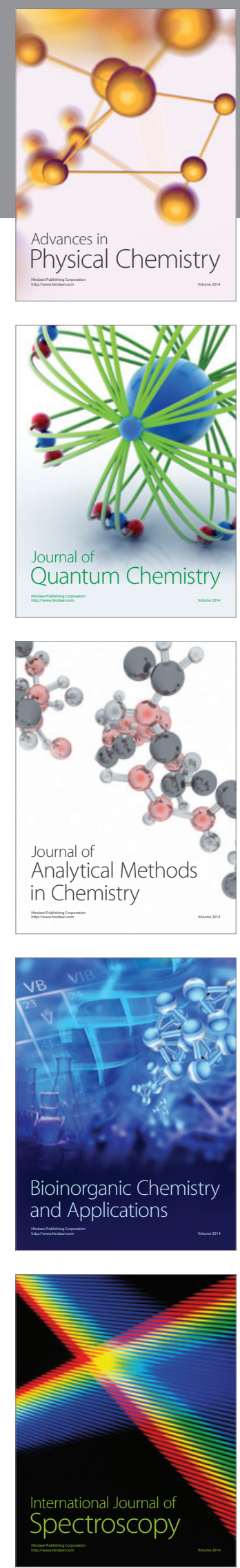